\title{
TWO-ELECTRON TRANSITION IN HOMOEPITAXIAL GaN LAYERS
}

\author{
A. Fiorek, J.M. Baranowski, A. Wysmolek, K. Pakula, M. Wojdak \\ Institute of Experimental Physics, Warsaw University \\ Hoża 69, 00-681 Warsaw, Poland
}

I. Grzegory and S. Porowski

High Pressure Research Center, Polish Academy of Sciences

Sokolowska 29, 01-142 Warsaw, Poland

It is shown that the luminescence mapping is a powerful method to help identify optical transitions. Two-electron transition was identified in the homoepitaxial GaN layer by this technique. It was found that the donor and acceptor bound exciton emissions are spatially displaced and show intensity maxima at different places of the epitaxial layer. It was also found that the $3.45 \mathrm{eV}$ line, suspected as "two-electron transition", follows exactly the donor bound exciton spatial distribution. Donor bound exciton recombines leaving the neutral donor in the excited $2 s$ state. Thus, $1 s-2 s$ excitation being equal to $22 \mathrm{meV}$ corresponds to $29 \mathrm{meV}$ hydrogenic donor binding energy. This is the first identification of the two-electron transition in GaN.

PACS numbers: 78.66.Fd, 78.55.Cr

Homoepitaxial $\mathrm{GaN}$ layers at low temperatures have intense emission spectra in the exciton region. In contrary to layers grown on sapphire, in which strain broadens optical transitions, the emission from the homoepitaxial samples consists of several narrow lines. Identification of processes responsible for observed optical transitions is important from the point of view of understanding of properties of a high quality homoepitaxial GaN layers.

One of experimental techniques which allows to identify optical transitions is the luminescence mapping. Observation of various optical transitions in a form of luminescence maps allows to find correlations between them and to identify them.

The GaN homoepitaxial layers were grown on a single crystal platelets of $\mathrm{GaN}$ by metalorganic chemical vapour deposition (MOCVD) in the same way as described in our earlier papers [1]. Luminescence of GaN was excited by $325 \mathrm{~nm}$ $\mathrm{He}-\mathrm{Cd}$ laser line and a system of moving mirrors allowed scanning of emission with a spatial resolution $20 \mu \mathrm{m}$. All results presented below correspond to measurements taken at $4.2 \mathrm{~K}$.

The typical luminescence spectrum of homoepitaxial GaN layer is shown in Fig. 1. The spectrum is dominated by a strong line due to donor bound exciton (DBE) at the energy $3.472 \mathrm{eV}$. At low energy side of the DBE peak there is a 


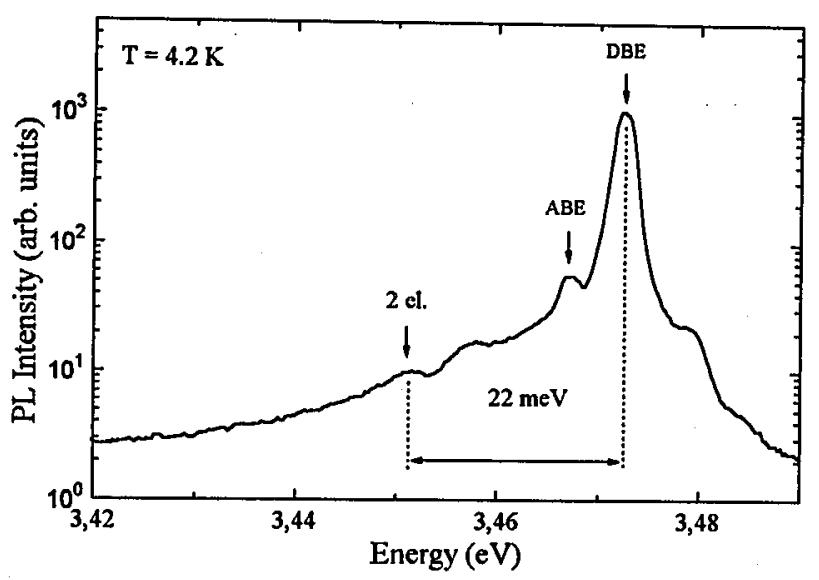

Fig. 1. The luminescence spectrum of homoepitaxial $\mathrm{GaN}$ in the exciton region, measured at $4.2 \mathrm{~K}$.

characteristic line due to acceptor bound exciton (ABE) at the energy $3.466 \mathrm{eV}$. These two main peaks were identified by magnetooptical experiment [2]. At high energy side of the DBE line there is a structure connected with free excitons, which was described previously [1]. At the energy $3.45 \mathrm{eV}$ there is a weak line, which was suggested to be connected to two-electron transition [3]. This emission line may arise from recombination of DBE with simultaneous excitation of neutral donor from $1 s$ to $2 s$ state. Experimental verification of the $1 s-2 s$ energy is important from the point of view of determination of the ionization energy of hydrogenic donor in GaN.

Mapping of emission at various energies may be helpful to find correlation between recombination processes taking place at these energies. In particular, one can look for correlation between $3.45 \mathrm{eV}$ emission and DBE or ABE line. The cross-sections through the luminescence maps on one of homoepitaxial layers for the $3.45 \mathrm{eV}, \mathrm{DBE}$ and ABE lines are shown in Fig. 2. One can see that the $3.45 \mathrm{eV}$ and DBE emission are correlated together. The most intense of ABE emissions is observed at the distance of about $100 \mu \mathrm{m}$ from the edge of the sample. On the other hand, the DBE and $3.45 \mathrm{eV}$ emissions are the most intense at the different place of layer's surface, at the distance of $700 \mu \mathrm{m}$ from the other edge of the sample. This proves that the $3.45 \mathrm{eV}$ emission is indeed connected with DBE. The $3.45 \mathrm{eV}$ peak is displaced by $22 \mathrm{meV}$ from the DBE line. There are two possible mechanisms, which may lead to the $22 \mathrm{meV}$ side band. One is a phonon replica of the DBE line and the other is two-electron transition connected with $1 s-2 s$ excitation of the shallow donor. If the $22 \mathrm{meV}$ side band is due to phonon one would expect that an even stronger replica, connected with $\mathrm{ABE}$, should be present. It has been found in $\mathrm{GaN}$ that phonons are more strongly coupled to $\mathrm{ABE}$ than to DBE [4]. However, even in Mg doped homoepitaxial layers where the ABE line is much stronger than the DBE one such replica is not observed. Therefore we may exclude the interpretation connected with phonon and we are left with 


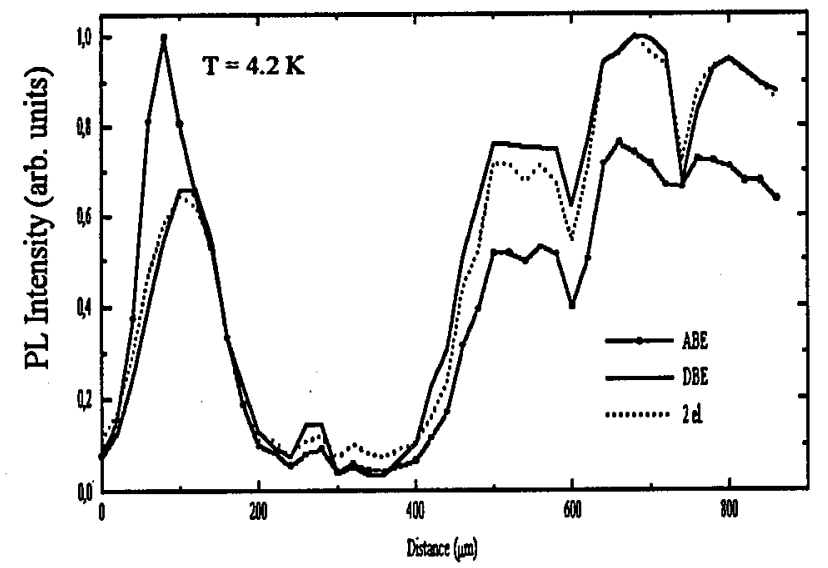

Fig. 2. The cross-sections of emission processes maps for: neutral acceptor bound exciton (circle), neutral donor bound exciton (solid line) and "two-electron transition" (dashed line).

the most likely interpretation that the two-electron transition is indeed observed at the energy $3.45 \mathrm{eV}$.

The energy of the $1 s-2 s$ transition equal to $22 \mathrm{meV}$ corresponds to the $29 \mathrm{meV}$ ionization energy of the shallow donor. This experimental value corresponds well with previous estimations: $41 \mathrm{meV} \mathrm{[5]} \mathrm{and} 29 \pm 6 \mathrm{meV}$ [6].

Finally, we would like to speculate on the origin of the donor present in homoepitaxial layers. It seems that oxygen is the donor impurity, which is present in all our epitaxial samples. Therefore we believe that the $29 \mathrm{meV}$ hydrogenic level is connected with oxygen impurity.

We gratefully acknowledge financial support from the Committee for Scientific Research grant No. 7 T08A 06110.

\section{References}

[1] K. Pakuła, A. Wysmołek, K.P. Korona, J.M. Baranowski, R. Stępniewski, I. Grzegory, M. Boćkowski, J. Jun, S. Krukowski, M. Wróblewski, S. Porowski, Solid State Commun. 97, 919 (1996).

[2] R. Stępniewski, A. Wysmołek, K. Pakuła, J.M. Baranowski, M. Potemski, G. Martinez, I. Grzegory, M. Wróblewski, S. Porowski, in: Proc. 23rd Int. Conf. on Physics of Semic., Berlin 1996, Eds. M. Scheffler, R. Zimmermann, World Scientific, Singapore 1996, p. 549.

[3] J.M. Baranowski, Z. Liliental-Weber, K. Korona, K. Pakuła, R. Stępniewski, A. Wysmołek, I. Grzegory, G. Nowak, S. Porowski, B. Monemar, P. Bergman, Mater. Res. Soc. Symp. Proc., 1997, in press.

[4] A. Wysmołek, P. Łomiak, J.M. Baranowski, K. Pakula, R. Stępniewski, K.P. Korona, I. Grzegory, M. Bockowski, S. Porowski, Acta Phys. Pol. A 90, 981 (1996).

[5] R. Dingle, M. llegems, Solid State Commun. 9, 175 (1971).

[6] O. Lagerstedt, B. Monemar, J. Appl. Phys. 45, 2266 (1974). 